\title{
Functional conservation despite structural divergence in ligand-responsive RNA switches
}

\author{
Mark A. Boerneke ${ }^{a}$, Sergey M. Dibrov ${ }^{a}$, Jing Gu ${ }^{a}$, David L. Wyles ${ }^{b}$, and Thomas Hermann ${ }^{a, c, 1}$ \\ ${ }^{a}$ Department of Chemistry and Biochemistry, ${ }^{b}$ Division of Infectious Diseases, Department of Medicine, and ${ }^{c}$ Center for Drug Discovery Innovation, University \\ of California, San Diego, La Jolla, CA 92093
}

Edited by Jennifer A. Doudna, University of California, Berkeley, CA, and approved October 2, 2014 (received for review July 31, 2014)

\begin{abstract}
An internal ribosome entry site (IRES) initiates protein synthesis in RNA viruses, including the hepatitis C virus (HCV). We have discovered ligand-responsive conformational switches in viral IRES elements. Modular RNA motifs of greatly distinct sequence and local secondary structure have been found to serve as functionally conserved switches involved in viral IRES-driven translation and may be captured by identical cognate ligands. The RNA motifs described here constitute a new paradigm for ligandcaptured switches that differ from metabolite-sensing riboswitches with regard to their small size, as well as the intrinsic stability and structural definition of the constitutive conformational states. These viral RNA modules represent the simplest form of ligand-responsive mechanical switches in nucleic acids.
\end{abstract}

IRES elements | translation regulation | RNA viruses | hepatitis C virus

nternal ribosome entry site (IRES) elements provide an alternative mechanism for translation initiation by directing the assembly of functional ribosomes directly at the start codon in a process that does not require $5^{\prime}$ cap recognition or ribosomal scanning and that is independent of many host initiation factors (1-4). The genomes of Flaviviridae and Picornaviridae contain elements that share similarity with the archetypical hepatitis $\mathrm{C}$ virus (HCV) IRES in overall domain organization, but not sequence or details of secondary structure (5). The HCV IRES adopts a complex architecture of four independently folding domains (Fig. 1A) (6). Domain II is nearly $100 \%$ conserved in clinical isolates (7) and has analogous counterparts in other viral IRES elements, all of which display some secondary structure similarity, but significant sequence variation in the subdomain IIa-like internal loop (Fig. 1B). Domain II has been shown to promote stable entry of $\mathrm{HCV}$ and classic swine fever virus (CSFV) mRNA at the decoding groove of the 40S subunit (8-10) and is required for initiation factor removal before ribosomal subunit joining (11), as well as adjustment of initiator tRNA orientation (12). The transition from initiation to elongation stages of translation depends critically on domain II (13). Recently, direct interaction of HCV domain II with initiator tRNA has been demonstrated (14). In HCV, subdomain IIa folds into an L-shaped motif (15) (Fig. 1C) that introduces a $90^{\circ}$ bend in domain II (16) and directs the IIb hairpin toward the E-site at the ribosomal subunit interface $(17,18)$.

The HCV IRES subdomain IIa is the target for viral translation inhibitors (Fig. $1 D$ ) that bind to the internal loop and block translation by capturing distinct conformational states of the RNA (7). Structure analysis revealed that benzimidazole inhibitors such as compound $\mathbf{1}(19,20)$ interact with an extended architecture of IIa in which the stems flanking the internal loop are coaxially stacked on both sides of the ligand-binding pocket (Fig. 1E) (21). In contrast, diaminopiperidine compounds such as 2 bind and lock the IIa RNA in a bent conformation that corresponds to the ligand-free state (22). Conformational capture of the subdomain IIa switch by ligands in solution was demonstrated by FRET experiments and established as a mechanism of IRES inhibition (23). On the basis of these findings, it was proposed that subdomain IIa may be the target for a cognate biological ligand whose adaptive recognition by the RNA motif may facilitate ribosome release from the IRES-bound complex (7).

Here, we have explored potential candidates for a cognate ligand of the subdomain IIa switch and investigated the structural and functional conservation of similar ligand responsive switch motifs in other IRES RNAs.

\section{Results}

Guanine Captures an Extended Conformation of the Subdomain Ila Switch. Cryoelectron microscopy studies of the HCV IRES bound to the ribosome reveal the bent domain II interacting with the $40 \mathrm{~S}$ subunit in a curved topology that would prevent the progression of the ribosome from initiation to elongation (17, 18). Conformational dynamics in the subdomain IIa RNA switch along with ligand capture of the extended state may facilitate removal of domain II from the ribosomal E site (21). Support for this hypothesis comes from recent cryo-EM studies that reveal major differences in the conformation of domain II in 40S-bound binary and 80S-bound initiation complexes of the HCV IRES (14). Synthetic benzimidazole translation inhibitors (compound 1), which capture an extended conformation of subdomain IIa, appear to be fortuitous ligands of this RNA motif (7). The nearperfect conservation across clinical isolates of residues in subdomain IIa (7), along with the adaptive formation of a deep pocket that encapsulates the small molecule reminiscent of riboswitches, led us to speculate about a cognate biological ligand. Binding of compound $\mathbf{1}$ to subdomain IIa depends critically on two hydrogen bonds to the Hoogsteen edge of a G-C base pair, closely resembling isosteric hydrogen bonding patterns

\section{Significance}

RNA viruses, including the human pathogenic hepatitis $C$ virus (HCV), use a structured untranslated region of their genome to hijack host cell ribosomes for the synthesis of viral proteins. These genome regions are termed internal ribosome entry site (IRES) elements and are encoded by distinct sequences in different viruses but share common functional RNA motifs. This study shows that viral IRES elements contain conformationally flexible RNA switches, whose state can be captured by the binding of a common ligand. Conformational switching plays a role in the function of the IRES elements. These new RNA switches are smaller than previously discovered "riboswitches" and may be the simplest form of ligand-responsive mechanical modules in nucleic acids.

Author contributions: M.A.B. and T.H. designed research; M.A.B., S.M.D., and J.G. performed research; D.L.W. contributed new reagents/analytic tools; M.A.B., S.M.D., and T.H. analyzed data; and M.A.B. and T.H. wrote the paper.

The authors declare no conflict of interest.

This article is a PNAS Direct Submission.

Data deposition: The atomic coordinates have been deposited in the Protein Data Bank, www.pdb.org (PDB ID codes 4P97 and 4PHY)

${ }^{1}$ To whom correspondence should be addressed. Email: tch@ucsd.edu.

This article contains supporting information online at www.pnas.org/lookup/suppl/doi:10 1073/pnas.1414678111/-/DCSupplemental. 


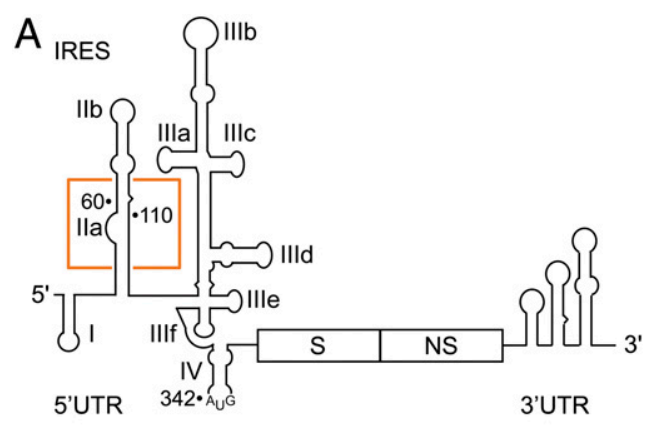

B
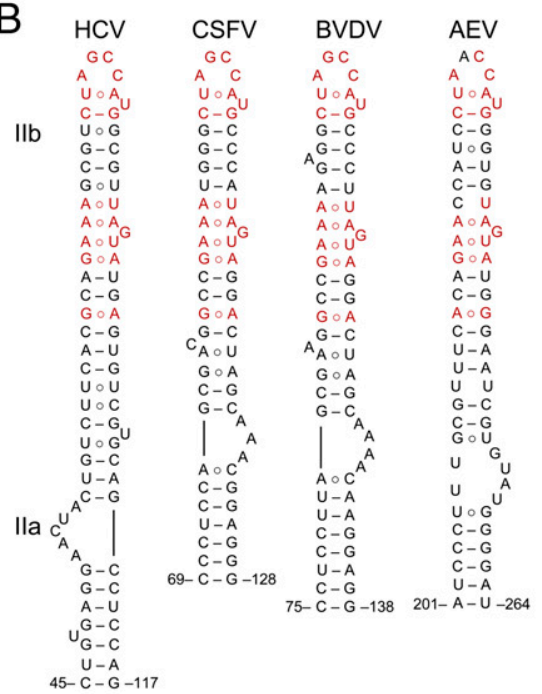

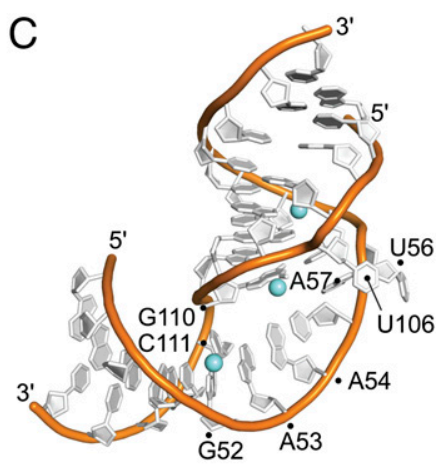

D

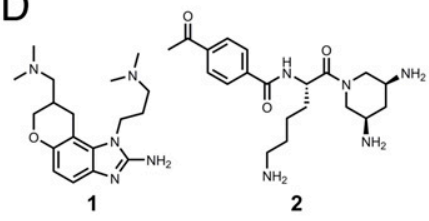

E

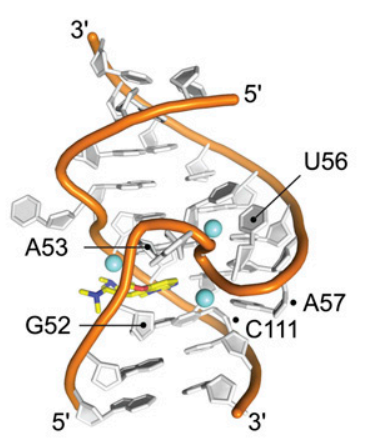

Fig. 1. Structures and ligands of viral IRES. (A) The IRES in the $5^{\prime}$ UTR of the HCV genome. The location of subdomain lla is highlighted by an orange box. The viral genome encodes structural (S) and nonstructural (NS) proteins and contains a structured 3' UTR. (B) Secondary structure predictions of domain II motifs in viral IRES elements from HCV and other flaviviruses, including CSFV and BVDV, as well as picornaviruses such as AEV and SVV. Non-WatsonCrick base pairs are indicated by the $O$ symbol. Sequence conservation is indicated in red. $(C)$ Crystal structure of the subdomain Ila RNA from HCV. (D) Benzimidazole (1) and diaminopiperidine (2) inhibitors of IRES-driven translation that target the HCV subdomain Ila. $(E)$ Crystal structure of the HCV subdomain Ila RNA in complex with inhibitor 1. observed for G-C pairs interacting with arginine in protein-RNA complexes $(24,25)$ and with guanosine in $\mathrm{C}-\mathrm{G} \circ \mathrm{G}$ triples $(26,27)$ (Fig. 2A).

Inspired by Yarus' classical study of arginine and $\mathrm{G}$ binding to self-splicing group I introns (28), we used a previously established FRET assay $(23,29)$ to test arginine, guanine, and their derivatives for binding to the subdomain IIa RNA. Conformational capture of the dye-labeled RNA switch (SI Appendix, Fig. $\mathrm{S} 1$ ) in the extended state leads to dose-dependent reduction of the FRET signal and concurrent increase of donor dye fluorescence resulting from diminished resonance energy transfer. The FRET assay has previously been used to demonstrate conformational capture of the IIa switch by benzimidazole ligands (23). Neither arginine nor its derivatives showed binding to the IIa RNA construct. In contrast, titration of guanine (3) elicited a dose-dependent FRET reduction indicative of ligand binding to the extended conformation of subdomain IIa (Fig. 2B). Although the binding activity of guanine was around $1 \mathrm{mM}\left(\mathrm{EC}_{50}\right.$ value), which is 300 times weaker than that of the benzimidazole $\mathbf{1}$, the concurrent increase of fluorescence from the $\mathrm{Cy} 3$ donor dye substantiated a selective ligand interaction, leading to conformational capture. Binding was specific for guanine, either as the base itself or in guanosine, whereas other nucleobases were inactive in the FRET assay (SI Appendix, Fig. S2 $A$ and $B$ ). The 2-aminopyrimidinone ring of guanine was essential for the interaction with the subdomain IIa RNA, as indicated by the binding activity of 2-aminoquinazolinone, which shares the pyrimidinone but not the imidazole heterocycle (compound 4, SI Appendix, Fig. S2C). The quinazolinone $\mathbf{4}$ bound the target about twofold tighter than guanine $\left(\mathrm{EC}_{50}\right.$ value, $\left.483 \mu \mathrm{M}\right)$, perhaps as a result of more favorable stacking interactions with the benzene ring in 4 .
To test for the effect of the guanine interaction on IRES function, we measured luciferase reporter expression levels from a bicistronic construct in an in vitro translation assay (29). The bicistronic construct allows measuring effects on IRES-driven translation of Renilla luciferase while also providing an internal control of a cap-initiated firefly luciferase (SI Appendix, Fig. S3). In agreement with the observed binding of guanine to subdomain IIa, the nucleobase selectively inhibited expression of the IRESdriven reporter (Fig. 2C). Translation inhibition required a higher concentration of guanine compared with 1 (SI Appendix, Fig. S4), again reflecting the weaker binding of guanine relative to the synthetic inhibitor. Inhibition of reporter expression was selective for guanine, as none of the other three nucleobases showed this effect (SI Appendix, Fig. S4).

Domain II-Like Motifs from Other IRES RNAs Are Unrelated in Sequence but Fold Similar to the HCV Archetype. IRES elements and their function in translation initiation differ fundamentally in distinct families of positive-sense RNA viruses. The IRES domains of flaviviruses such as HCV, CSFV, and bovine viral diarrhea virus (BVDV) recruit 40S subunits independent of eukaryotic initiation factors, whereas IRES elements of most picornaviruses require involvement of eukaryotic initiation factor 4 components (30). Despite the differences, RNA domains have been identified in the IRES elements of picornaviruses, including avian encephalomyelitis virus (AEV) and Seneca Valley virus (SVV) (31), which resemble analogous motifs in flaviviruses in overall secondary structure (5). RNA structures corresponding to the HCV domain II occur in the IRES elements of CSFV, BVDV, AEV, and SVV (Fig. 1B), as well as several other RNA viruses (5). Domain II-like RNAs share common motifs, 

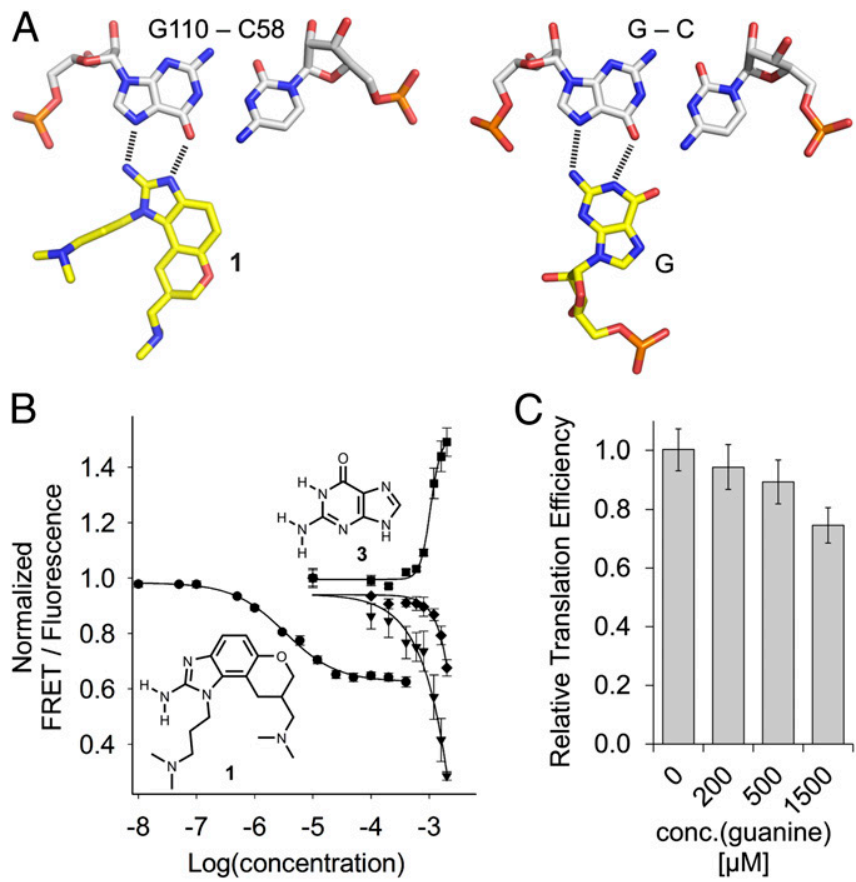

Fig. 2. Ligand binding to the HCV IRES. (A) Docking of the benzimidazole 1 at the C58-G110 base pair in the crystal structure of the HCV subdomain Ila target complex in comparison with the geometry of a C-G $\circ \mathrm{G}$ triple. (B) Titrations of Cy3/Cy5-labeled HCV subdomain Ila RNA with benzimidazole 1 and guanine 3. Curves show normalized FRET signal for $\mathbf{1}(\bullet)$ and guanine $(\boldsymbol{\nabla})$, as well as the normalized fluorescence signals of the donor Cy3 $(\square)$ and acceptor Cy5 $(\diamond)$ for guanine. Fitting of a single-site binding curve to the benzimidazole 1 FRET response gave an $\mathrm{EC}_{50}$ value for ligand binding of $3.4 \pm 0.3 \mu \mathrm{M}$. Because the FRET signal did not reach saturation in the guanine titration, affinity was estimated by fitting to the $\mathrm{Cy} 3$ emission, which resulted in an $\mathrm{EC}_{50}$ value for ligand binding of $1,050 \pm 69 \mu \mathrm{M}$ (guanine). (C) Effect of guanine on IRES-driven translation, as measured in an in vitro translation assay. Error bars in $B$ and $C$ represent \pm 1 SD calculated from triplicate experiments.

including a hairpin loop in subdomain IIb and a loop E motif (32), which are conserved in sequence and separation by base pairs (Fig. $3 A$ ). The internal loop of subdomain IIa shows no sequence conservation and differs both in size and location in the $5^{\prime}$ or $3^{\prime}$ proximal strand of the lower stem. NMR analysis of domain II from CSFV, which revealed a bent subdomain IIa (11), hinted at the structural similarity of subdomain IIa motifs despite their distinctions in sequence, size, and strand location.

To investigate whether subdomain IIa motifs from different viral IRES elements share the ability to adopt structurally similar bent architectures, we studied magnesium ion-induced folding of the RNAs by monitoring FRET. We had previously used the FRET assay to establish magnesium-dependent folding of the HCV subdomain IIa (23). We titrated dye-labeled constructs containing viral subdomain IIa motifs (SI Appendix, Fig. S1), which were initially free of metal ions, with increasing amounts of $\mathrm{Mg}^{2+}$ (Fig. $3 B$ ). FRET was not observed for any of the constructs in the absence of $\mathrm{Mg}^{2+}$ when the internal loop of subdomain IIa does not stably fold and the RNA adopts an extended conformation that places the dyes beyond the Förster radius. On the addition of $\mathrm{Mg}^{2+}$, the FRET signal appeared in a dosedependent fashion, indicating folding into a bent architecture for constructs of all viruses. Control RNA in which the internal loop was deleted did not show an increase of FRET (SI Appendix, Fig. $\mathrm{S} 2 D$ ). Effective $\mathrm{Mg}^{2+}$ concentrations for folding were of similar magnitude for all constructs, ranging from $\sim 130-600 \mu \mathrm{M}$ (Fig. $3 B$ ). Constructs that were more closely related with respect to overall secondary structure required similar amounts of magnesium for folding, delineating two distinct subtypes of subdomain IIa architectures (CSFV, BVDV, AEV: 131-219 $\mu \mathrm{M} \mathrm{Mg}^{2+}$; SVV, HCV: $579-598 \mu \mathrm{M} \mathrm{Mg}^{2+}$ ). Separation between these subtypes did not coincide with divisions between Flaviviridae and Picornaviridae.

To further explore the ability for conformational switching that subdomain IIa analogs may share in common with the HCV archetype motif, we used the FRET assay to test other viral motifs for interaction with the benzimidazole $\mathbf{1}$. Titration of the compound showed that ligand binding captured the subdomain IIa RNAs of CSFV, BVDV, and SVV in an extended state (SI Appendix, Fig. S2E), similar to that previously demonstrated for the HCV target (Fig. 2B), albeit requiring an 8-15-fold higher concentration of $\mathbf{1}$. The motif from AEV showed weaker interaction with the compound, and saturation of the ligand bound state was not achieved. Because subdomain IIa of SVV bound the benzimidazole tightest among the viral analogs, this RNA was also tested for its interaction with guanine. Similar to the subdomain IIa motif of HCV, the SVV analog was captured in an extended state by guanine at a comparable $\mathrm{EC}_{50}$ value of $990 \mu \mathrm{M}$ (SI Appendix, Fig. S2F).

Subdomain Ila RNA from the SVV IRES Element Adopts a $90^{\circ}$ Bent Fold. Because folding studies indicated that diverse viral subdomain IIa analogs adopt bent architectures, we attempted to elucidate the 3D structure of the RNAs. Crystal structures of two different constructs of the SVV subdomain IIa were determined. A high-resolution structure (1.86 $\AA$ ) was obtained for the internal loop (Fig. 4 and SI Appendix, Fig. S5), and a lower-resolution structure $(3.2 \AA)$ was obtained for an extended construct that included the region connecting to the loop E motif (SI Appendix, Fig. S6). Both structures showed identical architecture for the internal loop.

The SVV IRES subdomain IIa adopts a bent fold that arranges the flanking RNA helices at an angle of $90^{\circ}$ (Fig. $4 A$ and SI Appendix, Figs. S5 and S6). The overall architecture of subdomain II from SVV was nearly identical to that of the motif from HCV (SI Appendix, Fig. S7), despite differences in sequence and secondary structure. The folding of the bend in the SVV motif has no precedent in other RNA architectures. Except for the reverse Hoogsteen pair U369॰ A424, all base pairs adopt canonical Watson-Crick geometry, and all unpaired bases are
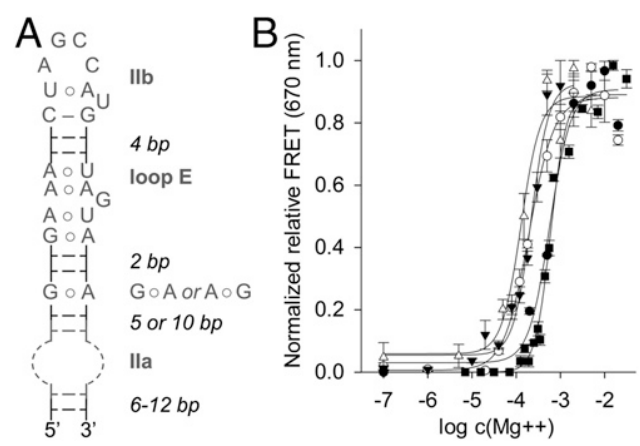

Fig. 3. Domain II-like RNAs in viral IRES elements. $(A)$ Consensus secondary structure of domain II analogs from viral IRES elements. The internal loop of subdomain lla consists of an unpaired stretch of nucleotides in either the 5 or $3^{\prime}$ proximal strand of the lower stem. $(B) \mathrm{Mg}^{2+}$-induced folding of viral subdomain Ila motifs. FRET signal from terminally Cy3/Cy5 labeled RNA constructs was monitored while increasing $\mathrm{Mg}^{2+}$ concentration. See SI Appendix, Fig. S1 for RNA construct structures. Fitting of dose-response curves resulted in $\mathrm{EC}_{50}$ values for $\mathrm{Mg}^{2+}$-induced folding at $598 \pm 25 \mu \mathrm{M}(\mathrm{HCV}, \square)$ (23), $196 \pm 21 \mu \mathrm{M}(\mathrm{CSFV}, \mathrm{O}), 219 \pm 19 \mu \mathrm{M}$ (BVDV, $\boldsymbol{\nabla}), 131 \pm 21 \mu \mathrm{M}(\mathrm{AEV}, \Delta)$, and $579 \pm 86 \mu \mathrm{M}(\mathrm{SVV}, \bullet)$. Error bars represent \pm 1 SD calculated from triplicate experiments. 

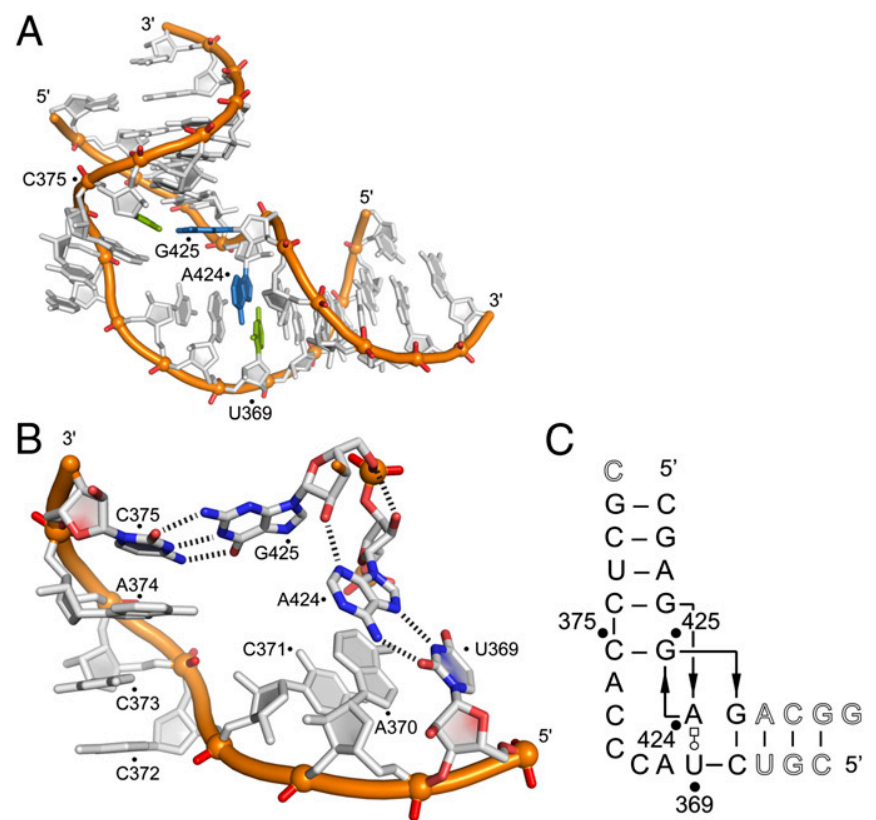

Fig. 4. Structure of the subdomain Ila RNA in the SVV IRES element. $(A)$ Crystal structure highlighting the perpendicular U369 $\circ$ A424 trans-WatsonCrick-Hoogsteen base pair and the Watson-Crick C375-G425 base pair. $(B)$ Detail view of the internal loop showing additional hydrogen bonds between $\mathrm{A} 424$ and $\mathrm{G} 425$ in the perpendicular base pairs $\left(\mathrm{N}{ }_{\mathrm{A} 424 \ldots .}{ }{ }^{\prime}{ }_{\mathrm{G} 425}\right.$ and

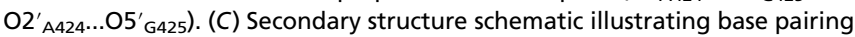
and consecutive stacking interactions. Nucleotides deviating from the SVV wild-type sequence are shown in outlined font.

continuously stacked on one of the flanking helices. The $\mathrm{U} 369 \circ \mathrm{A} 424$ and $\mathrm{C} 375-\mathrm{G} 425$ pairs are arranged orthogonally and provide the closing ends that interface the flanking helices to the internal loop (Fig. 4A). The perpendicular alignment of consecutive bases A424 and G425 is stabilized by hydrogen bonds involving the sugar backbone of these residues (Fig. 4B). Resulting from the orthogonal orientation of A424 and G425, the $\mathrm{U} 369 \circ \mathrm{A} 424$ and $\mathrm{C} 375-\mathrm{G} 425$ pairs form a cross-over motif, and strand directionality is locally inverted in the secondary structure (Fig. 4C).

Domain II-Like RNAs Are Interchangeable Functional Modules Despite Their Sequence Dissimilarity. To determine whether domain II analogs are autonomous RNA motifs that are functional as interchangeable modules, we investigated reporter expression from chimera IRES constructs in which the cognate HCV domain II was substituted by analogs from other viruses (SI Appendix, Fig. S3). Replacement of the HCV domain II by other viral analogs resulted in IRES proficient for translation initiation, except for the SVV domain, which does not carry a homologous hairpin sequence (Fig. 5A). These observations are in agreement with a previous report on translational activity for a similar $\mathrm{HCV}$ IRES chimera that had the domain II inserted from CSFV (33). Deletion of the subdomain IIa-like region in the chimera IRES elements, which ablates the bend in domain II, led to significant loss of function in all constructs (Fig. $5 A, \Delta \mathrm{II}$ ). Removal of two base pairs between the subdomain IIb hairpin loop and the conserved loop E motif, which affects both the distance and rotational orientation of the two motifs, resulted in a reduction of activity that was similar across different chimeras (Fig. $5 A ; \Delta 2 \mathrm{bp}$ ).

The availability of a crystal structure for the SVV subdomain IIa allowed us to determine a minimal architectural module in this RNA that corresponded to the bend in the HCV motif. Guided by superposition of RNA structures from both viruses, we substituted in the HCV IRES reporter construct the internal loop comprised of residues G52..U59/A109...C111 by the corresponding module from SVV consisting of C368...C376/ G423...G426 (Fig. 5B). Although the swapped RNA modules shared no sequence similarity, the resulting chimera IRES was fully proficient in translation initiation (Fig. $5 C$, construct $\mathrm{X}$ ). Even a small deviation from the structure-guided module swap, the introduction of a single base pair offset (construct P, SI Appendix, Fig. $\mathrm{S} 8$ ), resulted in a $50 \%$ loss in IRES activity (Fig. $5 C$ ). The chimera construct $\mathrm{X}$ also conferred full translation activity of HCV replicon in human cells $4 \mathrm{~h}$ after transfection, when viral translation occurs, but not yet replication (34) (Fig. 5D). However, expression activity from the chimera replicon was greatly diminished after $24 \mathrm{~h}$, when the cumulative reporter signal is expected to be dominated by replication. Apparently, the motif swap of subdomain IIa modules negatively affected communication between the 5'and 3' UTR, which has been implicated in viral replication $(35,36)$.

Replacement of the subdomain IIa motif in HCV by the corresponding analog from the CSFV IRES was performed to investigate the effect of relocating the internal loop from the $5^{\prime}$ to the $3^{\prime}$ proximal strand of the domain II lower stem. Secondary

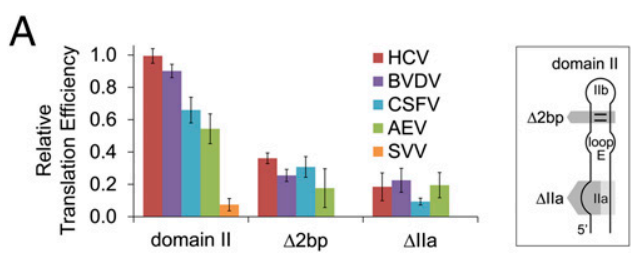

$\mathrm{B}$
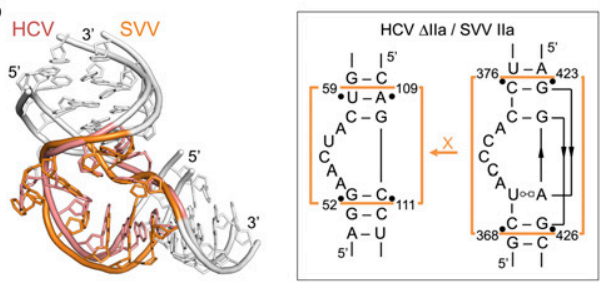

C

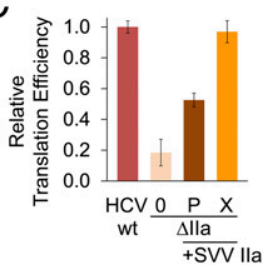

D

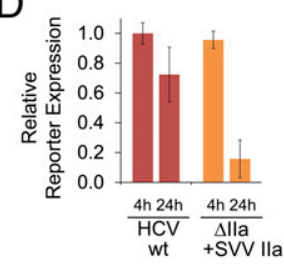

Fig. 5. Functional competence of chimera HCV IRES elements. $(A)$ Effect on in vitro translation of domain II replacement in the HCV IRES by corresponding motifs from other viral IRES elements and additional deletion of two base pairs between the hairpin loop IIb and the loop $E$ motif ( $\Delta 2 \mathrm{bp}$ ), or deletion of the internal loop Ila ( $\Delta$ lla). See SI Appendix, Fig. S3 for chimera structure and sequences. $(B)$ Construction of a chimera $X$ (orange) by an exact structural motif swap based on superimposition of the subdomain lla crystal structures from HCV (red/white) and SVV (orange/white). (C) Effect on in vitro translation of subdomain Ila replacement in the HCV IRES by the corresponding motif from SVV (chimera $X$, outlined in $B$ ). The chimera $P$ (brown) is a control construct that contains a subdomain lla swap with a one base pair offset relative to the $X$ construct (SI Appendix, Fig. S8). HCV IRES wild-type and subdomain lla deletion $(\Delta \mathrm{lla}, 0)$ are shown as controls. Translation efficiencies were normalized to the cap-driven expression in bicistronic dual reporter constructs. (D) Function of the HCVISVV IRES chimera $X$ in replicon-transfected human cells. Reporter expression was measured 4 and $24 \mathrm{~h}$ after transfection, with the subgenomic replicon RNA carrying the IRES subdomain Ila replaced by the corresponding motif from SVV. Error bars represent \pm 1 SD calculated from triplicate experiments, except for in $D$, where triplicates of three biological replicates (nine values) were used. 
structure-guided swapping of the internal loop modules did not result in a translation-proficient IRES. An active chimera was obtained only after shortening the distance by 5 base pairs between the subdomain IIa internal loop and the conserved loop E, in combination with relocating a bulged-out pyrimidine from the $3^{\prime}$ to the $5^{\prime}$ proximal strand (SI Appendix, Fig. S9). These changes were motivated by the observation that in domain II analogs that carry the internal loop in the $3^{\prime}$ proximal strand, the distance to the loop E motif is shorter by 5 base pairs, corresponding to a half turn of an A-form RNA helix. A bulged-out pyrimidine, separated by 4 base pairs above the subdomain IIa internal loop, is found consistently in the strand opposite to the internal loop (Fig. $1 B$ ). These observations provided the basis for a topological model of the modular domain II architecture in viral IRES elements (Fig. $6 A$ ).

\section{Discussion}

The highly conserved subdomain IIa in the HCV IRES had previously been established as an RNA conformational switch that is able to convert from a ligand-free bent state to a ligandbound extended conformation (7). The bent RNA fold is stabilized by magnesium ions and ensures the correct positioning of the IRES on the 40S ribosomal subunit and docking of domain II at the E site $(17,18)$. Removal of domain II from the ribosome during translation initiation may be topologically achieved by a conformational change in subdomain IIa transitioning from the bent to the extended state (21). Recent cryo-EM studies confirm the conformational flexibility of domain II through comparison of 40S-bound binary and 80S-bound initiation complexes of the HCV IRES (14). Motivated by the discovery of a riboswitch-like binding site for benzimidazole translation inhibitors (19) in the extended form of subdomain IIa (21), we hypothesized that a cognate ligand may serve as the biological actuator of the RNA switch that ultimately facilitates ribosomal release from the IRES. Inspection of the crystal structure previously determined for an inhibitor complex of subdomain IIa suggested that benzimidazole derivatives might be fortuitous ligands that exploit a recognition site for guanine. Here, we used a FRET assay to demonstrate binding and capture of the subdomain IIa RNA in an extended conformation by guanine and guanosine. Guanine binding to the subdomain IIa target led to inhibition of IRES-driven translation, albeit requiring a $\sim 300$-fold higher concentration compared with potent benzimidazole derivatives. Structural comparison shows that the guanine base may engage in similar interactions with subdomain IIa as those observed for benzimidazole inhibitors. The resulting arrangement corresponds to the interaction of guanosine with a Watson-Crick G-C pair in a geometry that accounts for one of the most frequently occurring base triples in RNA folds (27).

Modeling of guanine in the ligand-binding pocket of the benzimidazole-RNA crystal structure revealed suboptimal stacking by the nucleobase on neighboring residues (SI Appendix, Fig. S10). Better stacking is achieved with the larger quinazolinone 4 , which has a $\sim$ twofold higher affinity for the RNA target than guanine (Fig. $2 B$ ). The smaller size in combination with the neutral character of the ligand may explain the weaker binding affinity of guanine compared with benzimidazole inhibitors. The nucleobase as a putative biological ligand of the subdomain IIa RNA switch may function as a transient actuator that readily dissociates from the IRES, unlike the benzimidazoles, which arrest the IRES topology and thereby inhibit translation initiation. It is conceivable that a guanosine in an RNA sequence such as ribosomal RNA or the viral genome emerging from the ribosome may serve as a trigger facilitating IRES release from the ribosome. Docking of IRES fragment crystal structures to a cryoEM map of an IRES-40S ribosomal complex revealed a cluster of guanosines from the central domain pseudoknot and domain IV residing in a single-stranded stretch upstream of the viral initiation codon and located in close proximity to subdomain IIa (37). Involvement of a guanosine from the viral genome as a trigger for the

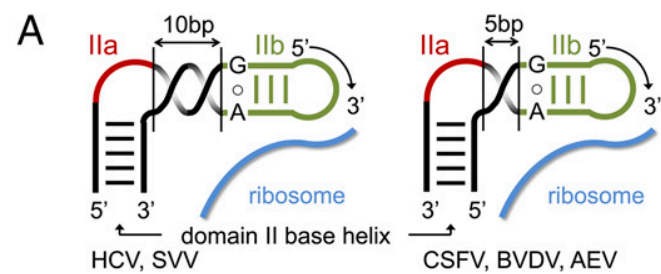

$B$

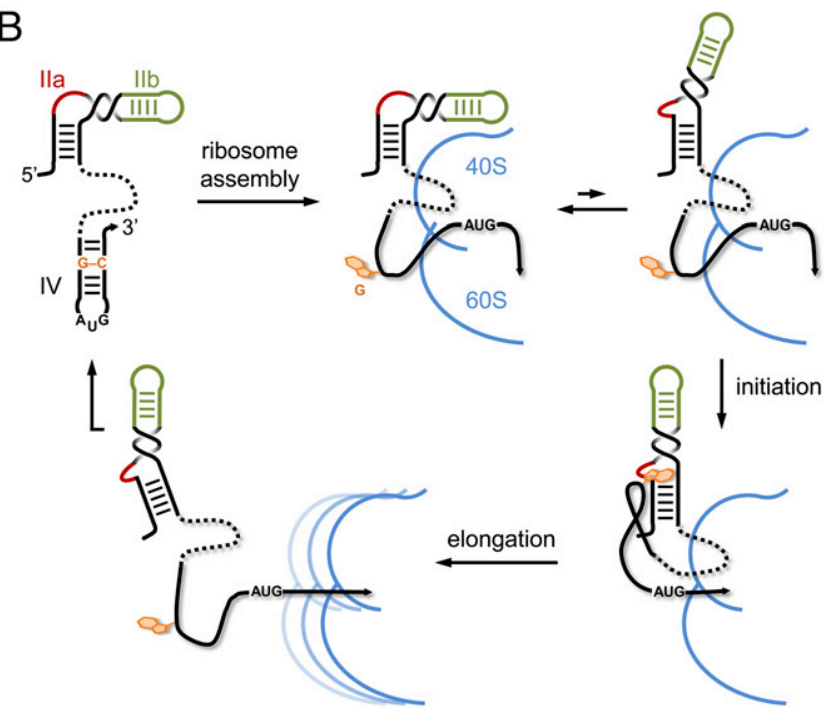

Fig. 6. Model for the modular domain II architecture in viral IRES elements and its participation in IRES regulation. (A) For the correct positioning of the conserved hairpin loop IIb at the $40 \mathrm{~S}$ ribosomal subunit, the bent internal loop Ila module has to be located in the $5^{\prime}$ or $3^{\prime}$ strand of the base helix, depending on the number of base pairs in the spacer segment between lla and IIb. A spacer of 10 base pairs, as in the IRES elements of HCV and SVV, corresponds to a full turn of an A-form RNA helix, whereas a spacer of 5 base pairs provides a half turn. (B) Proposed mechanism for the regulation of IRES activity by the subdomain Ila switch through an interaction with a $G$ residue from domain IV. Ribosome binding of the IRES entails melting of the domain IV hairpin to provide access of the viral start codon to the decoding site, which in turn unmasks the trigger $G$ residue that facilitates IRES release during initiation by capture of the Ila extended state. After the ribosome transitions to elongation, the unbound IRES may refold to the original state.

subdomain IIa switch would capitalize on a locally high concentration of the ligand as a part of the same RNA strand. In contrast, participation of cellular guanine or guanosine is unlikely because of their low physiological concentration of around 97 and $0.9 \mu \mathrm{M}$, respectively (38). GTP, although present in human cells at a higher level $(\sim 300 \mu \mathrm{M})$, is a less suitable ligand for the IIa target because of electrostatic repulsion by the phosphate groups.

Regulation of the HCV IRES activity by the subdomain IIa switch through interaction in cis with a guanosine from domain IV would provide an efficient autoregulatory mechanism in which the trigger $\mathrm{G}$ residue is sequestered in a hairpin loop of the unbound IRES to allow ribosome assembly supported by the bent IIa RNA motif. Placement of the viral start codon at the decoding site requires melting of the domain IV hairpin, which in turn unmasks the trigger $\mathrm{G}$ residue that facilitates IRES release during initiation through capture of the IIa extended state (Fig. $6 B$ ).

The discovery of the IRES subdomain IIa as a ligandresponsive RNA switch in HCV raised the question of whether corresponding motifs in other viruses adopt analogous folds whose conformational state may be captured by ligand binding. Here, we demonstrated that RNA motifs from several flaviviruses and picornaviruses fold into magnesium-stabilized bent architectures similar to the $\mathrm{HCV}$ archetype, despite their sequence 
dissimilarity (Fig. 3). Earlier NMR studies of domain II from CSFV performed at lower salt concentration already showed a bent structure for this RNA (11). The crystal structure of the internal loop motif from SVV (Fig. 4) revealed a fold that is overall identical to the HCV subdomain IIa despite the fact that the two motifs share little sequence or local secondary structure similarity (SI Appendix, Fig. S8). Analogous subdomain IIa RNAs from other viral IRESs interact with the benzimidazole 1, as well as guanine, to be captured in an extended conformation (SI Appendix, Fig. S2 $E$ and $F$ ). Binding of $\mathbf{1}$ to the subdomain IIa RNAs from other viruses was $\sim 10$-fold weaker than the affinity measured for the HCV target, perhaps reflecting the extensive optimization of the benzimidazole derivative for inhibition of the HCV IRES $(19,39)$.

The analogies between viral subdomain IIa RNA motifs extend beyond the similarity of static structure and the ability to adopt two distinct conformational states. Domain swap experiments in which crystal structure information was used to precisely replace the subdomain IIa in the HCV IRES by the corresponding motif from SVV demonstrated that biological function is completely conserved between these intrinsically distinct RNA building blocks (Fig. $5 B$ and $C$ ). HCV-SVV chimera IRES elements were fully functional both in vitro and in replicon-infected cells. Even the structurally less accurate replacement of the whole domain II in the HCV RNA with analogous motifs from BVDV, CSFV, and AEV yielded functional chimera IRES elements (Fig. $5 A$ ). We conclude that subdomain IIa motifs of greatly distinct sequence and local secondary

1. Plank TD, Kieft JS (2012) The structures of nonprotein-coding RNAs that drive internal ribosome entry site function. Wiley Interdiscip Rev RNA 3(2):195-212.

2. Hellen CU, Pestova TV (1999) Translation of hepatitis C virus RNA. J Viral Hepat 6(2):79-87.

3. Ji H, Fraser CS, Yu Y, Leary J, Doudna JA (2004) Coordinated assembly of human translation initiation complexes by the hepatitis $C$ virus internal ribosome entry site RNA. Proc Natl Acad Sci USA 101(49):16990-16995.

4. Otto GA, Puglisi JD (2004) The pathway of HCV IRES-mediated translation initiation Cell 119(3):369-380.

5. Hellen CU, de Breyne S (2007) A distinct group of hepacivirus/pestivirus-like internal ribosomal entry sites in members of diverse picornavirus genera: Evidence for modular exchange of functional noncoding RNA elements by recombination. J Viro/ 81(11):5850-5863.

6. Kieft JS, et al. (1999) The hepatitis C virus internal ribosome entry site adopts an iondependent tertiary fold. J Mol Biol 292(3):513-529.

7. Dibrov SM, et al. (2014) Hepatitis $C$ virus translation inhibitors targeting the internal ribosomal entry site. J Med Chem 57(5):1694-1707.

8. Pestova TV, Shatsky IN, Fletcher SP, Jackson RJ, Hellen CU (1998) A prokaryotic-like mode of cytoplasmic eukaryotic ribosome binding to the initiation codon during in ternal translation initiation of hepatitis $C$ and classical swine fever virus RNAs. Genes Dev 12(1):67-83.

9. Kolupaeva VG, Pestova TV, Hellen CU (2000) Ribosomal binding to the internal ribosomal entry site of classical swine fever virus. RNA 6(12):1791-1807.

10. Filbin ME, Kieft JS (2011) HCV IRES domain Ilb affects the configuration of coding RNA in the 40 S subunit's decoding groove. RNA 17(7):1258-1273.

11. Locker N, Easton LE, Lukavsky PJ (2007) HCV and CSFV IRES domain II mediate elF2 release during 80 S ribosome assembly. EMBO J 26(3):795-805.

12. Filbin ME, Vollmar BS, Shi D, Gonen T, Kieft JS (2013) HCV IRES manipulates the ribosome to promote the switch from translation initiation to elongation. Nat Struct Mol Biol 20(2):150-158.

13. Pestova TV, de Breyne S, Pisarev AV, Abaeva IS, Hellen CU (2008) elF2-dependent and eIF2-independent modes of initiation on the CSFV IRES: A common role of domain II. EMBO J 27(7):1060-1072.

14. Yamamoto $\mathrm{H}$, et al. (2014) Structure of the mammalian $80 \mathrm{~S}$ initiation complex with initiation factor 5B on HCV-IRES RNA. Nat Struct Mol Biol 21(8):721-727.

15. Dibrov SM, Johnston-Cox H, Weng YH, Hermann T (2007) Functional architecture of HCV IRES domain II stabilized by divalent metal ions in the crystal and in solution. Angew Chem Int Ed Engl 46(1-2):226-229.

16. Lukavsky PJ, Kim I, Otto GA, Puglisi JD (2003) Structure of HCV IRES domain II de termined by NMR. Nat Struct Biol 10(12):1033-1038.

17. Spahn CM, et al. (2001) Hepatitis $C$ virus IRES RNA-induced changes in the conformation of the 40s ribosomal subunit. Science 291(5510):1959-1962.

18. Boehringer D, Thermann R, Ostareck-Lederer A, Lewis JD, Stark H (2005) Structure of the hepatitis $C$ virus IRES bound to the human 80 S ribosome: Remodeling of the HCV IRES. Structure 13(11):1695-1706.

19. Seth PP, et al. (2005) SAR by MS: Discovery of a new class of RNA-binding small molecules for the hepatitis $C$ virus: Internal ribosome entry site IIA subdomain. $J$ Med Chem 48(23):7099-7102.

20. Parker MA, Satkiewicz E, Hermann T, Bergdahl BM (2011) An efficient new route to dihydropyranobenzimidazole inhibitors of HCV replication. Molecules 16(1):281-290. structure may serve as functionally conserved RNA conformational switches that are involved in viral IRES-driven translation and may be captured by highly similar or even identical ligands. We propose that the biological capture ligand shared between the different viral RNA motifs is likely a guanosine.

The functionally conserved subdomain IIa motifs constitute a new paradigm for ligand-captured RNA switches that differ from metabolite-sensing riboswitches with regard to their small size, as well as the intrinsic stability and structural definition of the constitutive conformational states. These viral RNA modules represent the simplest form of ligand-responsive mechanical switches in nucleic acids.

\section{Materials and Methods}

Preparation of RNA constructs is outlined in the SI Appendix, Supporting Materials and Methods. FRET folding and compound screening experiments were performed as described earlier (29). The in vitro transcription-translation assay was performed using HCV bicistronic luciferase constructs, as previously reported (40). HCV replicon testing followed procedures outlined earlier $(23,41)$. Experimental details for these methods, as well as the crystallization and structure determination of SVV subdomain Ila RNA, are described in the SI Appendix, Supporting Materials and Methods.

ACKNOWLEDGMENTS. M.A.B. was supported by a Graduate Assistance in Areas of National Need fellowship from the US Department of Education. Support for this project was provided by University of California, San Diego, Academic Senate Grant RM069B, for the Biomolecule Crystallography Facility by the National Institutes of Health (Grant OD011957), and for the NMR facility by the National Science Foundation (Chemistry Research Instrumentation and Facilities Program Grant CHE-0741968).

21. Dibrov SM, et al. (2012) Structure of a hepatitis $C$ virus RNA domain in complex with a translation inhibitor reveals a binding mode reminiscent of riboswitches. Proc Natl Acad Sci USA 109(14):5223-5228.

22. Carnevali M, Parsons J, Wyles DL, Hermann T (2010) A modular approach to synthetic RNA binders of the hepatitis $C$ virus internal ribosome entry site. ChemBioChem 11(10):1364-1367.

23. Parsons J, et al. (2009) Conformational inhibition of the hepatitis $C$ virus internal ribosome entry site RNA. Nat Chem Biol 5(11):823-825.

24. Puglisi JD, Chen L, Frankel AD, Williamson JR (1993) Role of RNA structure in arginine recognition of TAR RNA. Proc Natl Acad Sci USA 90(8):3680-3684.

25. Kondo J, Westhof E (2011) Classification of pseudo pairs between nucleotide bases and amino acids by analysis of nucleotide-protein complexes. Nucleic Acids Res 39(19): 8628-8637.

26. Walberer BJ, Cheng AC, Frankel AD (2003) Structural diversity and isomorphism of hydrogen-bonded base interactions in nucleic acids. J Mol Bio/ 327(4):767-780.

27. Abu Almakarem AS, Petrov Al, Stombaugh J, Zirbel CL, Leontis NB (2012) Comprehensive survey and geometric classification of base triples in RNA structures. Nucleic Acids Res 40(4):1407-1423.

28. Yarus M (1988) A specific amino acid binding site composed of RNA. Science 240(4860):1751-1758.

29. Zhou S, Rynearson KD, Ding K, Brunn ND, Hermann T (2013) Screening for inhibitors of the hepatitis C virus internal ribosome entry site RNA. Bioorg Med Chem 21(20):6139-6144.

30. Pestova TV, et al. (2001) Molecular mechanisms of translation initiation in eukaryotes. Proc Natl Acad Sci USA 98(13):7029-7036

31. Willcocks MM, et al. (2011) Structural features of the Seneca Valley virus internal ribosome entry site (IRES) element: A picornavirus with a pestivirus-like IRES. J Virol 85(9):4452-4461.

32. Leontis NB, Westhof $E$ (1998) A common motif organizes the structure of multi-helix loops in $16 \mathrm{~S}$ and $23 \mathrm{~S}$ ribosomal RNAs. J Mol Biol 283(3):571-583.

33. Reusken CB, Dalebout TJ, Eerligh P, Bredenbeek PJ, Spaan WJ (2003) Analysis of hepatitis $C$ virus/classical swine fever virus chimeric 5'NTRs: Sequences within the hepatitis C virus IRES are required for viral RNA replication. J Gen Virol 84(Pt 7):1761-1769.

34. Blight KJ, McKeating JA, Marcotrigiano J, Rice CM (2003) Efficient replication of hepatitis C virus genotype 1a RNAs in cell culture. $J$ Virol 77(5):3181-3190.

35. Shi ST, Lai MMC (2006) HCV 5' and 3'UTR: When Translation Meets Replication. Hepatitis C Viruses: Genomes and Molecular Biology, ed Tan SL (Horizon Bioscience, Norfolk).

36. Romero-López C, Berzal-Herranz A (2009) A long-range RNA-RNA interaction between the $5^{\prime}$ and $3^{\prime}$ ends of the HCV genome. RNA 15(9):1740-1752.

37. Berry KE, Waghray S, Mortimer SA, Bai Y, Doudna JA (2011) Crystal structure of the HCV IRES central domain reveals strategy for start-codon positioning. Structure 19(10):1456-1466.

38. Traut TW (1994) Physiological concentrations of purines and pyrimidines. Mol Cell Biochem 140(1):1-22.

39. Seth PP, Jefferson EA, Griffey RH, Swayze EE (2004) Benzimidazoles and analogs thereof as antivirals. World Patent Appl WO2004050035A3.

40. Brunn ND, Garcia Sega E, Kao MB, Hermann T (2012) Targeting a regulatory element in human thymidylate synthase mRNA. ChemBioChem 13(18):2738-2744.

41. Wyles DL, Kaihara KA, Vaida F, Schooley RT (2007) Synergy of small molecular inhibitors of hepatitis C virus replication directed at multiple viral targets. J Virol 81(6):3005-3008. 